\title{
Transaction
}

\section{Reaction of Nylon 6 Fiber with Chitosan using Cyanuric Chloride}

\author{
Takeru Ohe, Yurika Yoshimura, and Ikuo Abe
}

\author{
Osaka Municipal Technical Research Institute, Osaka 536-8553, Japan
}

\begin{abstract}
Combination of reaction of nylon 6 fiber with cyanuric chloride and subsequent reaction with chitosan gave nylon 6 fiber bonding chitosan chemically. Especially, the latter reaction improved not only the hygroscopicity but also dyeing ability for reactive dyes. Furthermore, when a one-pot method using cationic surfactant LTAC was also investigated, above two reactions could proceed simultaneously and obtained nylon 6 fiber showed the highest hygroscopicity in this work.
\end{abstract}

(Received 19 December, 2006 ; Accepted 2 March, 2007)

\section{Introduction}

Nylon 6 fibers have been widely used as garments or industrial textiles secondary to PET fibers and polyacrylic fibers in synthetic fibers, because of their low costs and their many good fiber properties such as high mechanical strength, good dyeing ability, and good dimensional stability and so on. Furthermore, recent reports on the chemical recycle of nylon 6 using supercritical water have been expected to expand their market share from the point of a recyclable synthetic fiber $[1,2]$. However, nylon 6 fibers also have many problems such as low hygroscopicity and high electrostatic propensity as same as other synthetic fibers. If terminal amino groups or carboxyl groups of nylon fibers can react with hydrophilic compounds with reactive groups such as cyanuric groups, obtained nylon fibers become a new comfortable synthetic fiber to solve above problems. That is, such fibers can adsorb excessive moisture in cloths in summer or disperse static electricity generating on cloths in winter. In the present time, there are no reports on high hygroscopic nylon fibers using reactions with their terminal groups, because few these groups exist on the fiber surface enough to react with such functional compounds.

Meanwhile, chitosan is natural polymer composed of D-glucosamine units, which can be mass-produced from shells of crustaceans such as crabs or shrimps. For reasons of high safety of chitosan for human body or natural environment, various products containing chitosan such as diet foods, health foods, cosmetics, and adhesive agents for building materials have been on the market recently. However, a large amount of chitosan has still remained as wastes due to serious problems of the very low solubility into almost all solvents including water. Generally, commercial chitosan with large molecular weight can be soluble only in weak acid water solution such as aqueous acetic acid. In some reports on fiber processings, chitosan can also be used as antibacterial reagents or dyeing improvement reagents, where chitosan was fixed on fibers using crosslinking reactions of chitosan, or mixed into fibers without chemical bonds between chitosan and fibers [3-6]. Therefore, the introduced chitosan were gradually off from the fiber surface by laundry using water. Recently, an interesting method was reported to combine chitosan with cotton fibers chemically [7], in which the following two-step reactions were used: (1) reaction of cyanuric chloride
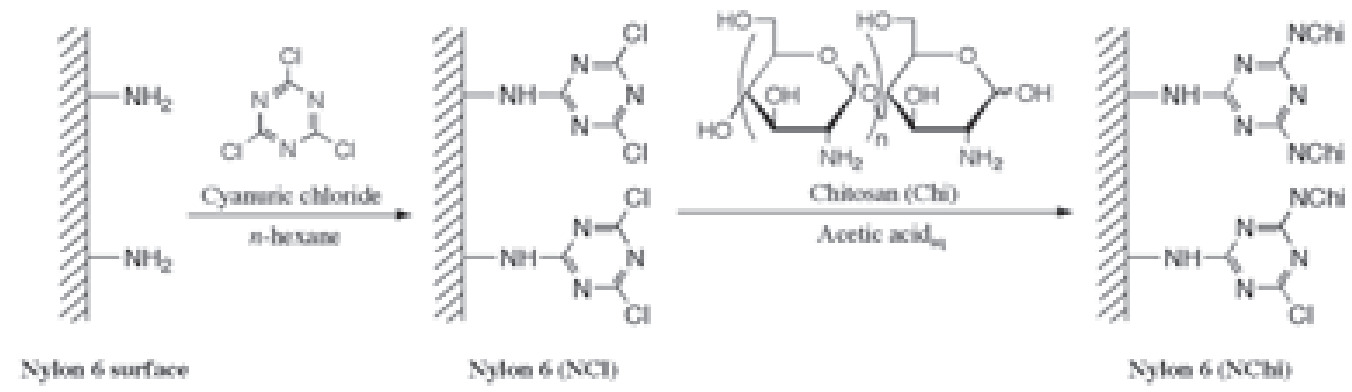

(a)

Fig. 1 Surface modification of nylon 6 fiber by cyanuric chloride and chitosan (2-step reaction). 
with hydroxyl groups of the cotton fibers (introduction of reactive cyanuric groups), (2) reaction of the cyanuric groups introduced on the cotton fibers with chitosan in the buffer water solution including acetic acid.

In our laboratory, chemical modifications of hydrophobic PET fibers using hydrophilic sugars or their derivatives have been investigated for several years [810]. Introduction of sugar moieties on PET fiber resulted in improvement of both the hygroscopicity and the dyeing ability. Our most recent report showed that PET fiber having amino groups could react with various sugars including sugars of low reactivity such as trehalose via introduction of the reactive cyanuric groups described above [10]. This method can be also easily applied to various fibers having amino groups such as nylon or other amide fibers. In this paper, we report 2-step reaction including both the reaction of nylon 6 fibers with cyanuric chloride and subsequent reaction with chitosan as figured in Fig. 1. Reacted nylon 6 fibers can be expected as a new type of synthetic fibers showing not only high hygroscopic property and the low electrostatic propensity but also high antibacterial activity arising from chitosan. Furthermore, a one-pot method using surfactants is also investigated in this paper, where both the reaction of nylon 6 fibers with cyanuric chloride and the reaction with chitosan can be expected to proceed simultaneously in the aqueous solution.

\section{Experimental}

\subsection{Reagents and nylon 6 fabric}

Cyanuric chloride, $n$-hexane, chitosan from crab shell, acetic acid, lauryltrimethylammonium chloride (LTAC), and sodium dodecyl sulfate (SDS) were purchased from Nacalai Tesque Co., Japan. Acid dye (C.I. Acid Red 52) and reactive dye (C.I. React Red 21) were purchased from Nagase Colors \& Chemicals Co., Japan. These reagents and dyes were used without further purification. Nylon 6 fabric (taffeta, standard white) was purchased from Shikisensha Co., Japan. The Nylon 6 fabric was washed thoroughly with hexane, methanol, and distilled water and then dried overnight before the following experiments.

\subsection{Surface modification of nylon 6 fabric}

\subsubsection{Reaction of nylon 6 fabric with cyanuric chloride}

A mixture of cyanuric chloride $1.84 \mathrm{~g}$ and hexane $150 \mathrm{ml}$ was stirred vigorously in $200 \mathrm{ml}$ flask at reflux condition until the solution was suspended. After nylon 6 fabric $(4.0 \mathrm{~cm} \times 4.0 \mathrm{~cm}$ for measurement of hygro- scopicity) was added there, the solution was stirred for a given time keeping at the same temperature. The obtained nylon $6(\mathrm{NCl})$ fabric was washed thoroughly with hexane, cooled methanol, and dried at room temperature.

\subsubsection{Reaction of nylon 6 ( $\mathrm{NCl}$ ) fabric with chitosan}

$100 \mathrm{ml}$ acetic acid water solution containing the chitosan $([\mathrm{AcOH}]=0-10 \mathrm{vol} \%$, [chitosan $]=0-10 \mathrm{~g} / \mathrm{l})$ was kept at the constant temperature $\left(25,40,60\right.$, and $\left.80{ }^{\circ} \mathrm{C}\right)$ in a $150 \mathrm{ml}$ flask, and then the nylon $6(\mathrm{NCl})$ fabric was placed in it. After the constant period, nylon 6 (NChi) fabric was picked out, washed thoroughly with hot distilled water, and dried at room temperature.

\subsubsection{Reaction of chitosan with cyanuric chloride}

A mixture of cyanuric chloride $0.92 \mathrm{~g}$ and hexane $100 \mathrm{ml}$ was stirred vigorously in a $150 \mathrm{ml}$ flask at reflux condition until the solution was suspended. After chitosan $0.10 \mathrm{~g}$ was added in the flask, the solution was stirred for $2 \mathrm{hr}$ keeping in refluxing hexane. The obtained mixture (chitosan $(\mathrm{NCl})$ ) of chitosan having cyanuric groups partly was filtered, and washed thoroughly with hexane, cooled methanol, and dried at room temperature. These products were used without further purification in the following experiments.

\subsubsection{Reaction of nylon 6 fabric with chitosan (NCl)}

$100 \mathrm{ml}$ acetic acid water solution (5.0vol\%) involving the chitosan $(\mathrm{NCl}) 0.10 \mathrm{~g}$ was stirred in a 150 $\mathrm{ml}$ flask at $80{ }^{\circ} \mathrm{C}$, and then nylon 6 fabric $(4.0 \mathrm{~cm} \mathrm{x} 4.0$ $\mathrm{cm})$ was placed in it. After the constant period, nylon 6 (NChi) fabric was picked out, washed thoroughly with hot distilled water, and dried at room temperature.

\subsubsection{Reaction of nylon 6 fabric with cyanuric chloride and chitosan (one-pot method)}

$100 \mathrm{ml}$ water solution of $5.0 \mathrm{vol} \%$ acetic acid containing the chitosan $0.10 \mathrm{~g}$, cyanuric chloride $0.92 \mathrm{~g}$, and LTAC $2.68 \mathrm{~g}$ was prepared in a $150 \mathrm{ml}$ flask, and was stirred keeping at $80{ }^{\circ} \mathrm{C}$. After the mixture became clear, nylon 6 fabric $(4.0 \mathrm{~cm} \times 4.0 \mathrm{~cm})$ was placed in it. After the constant period, the nylon 6 (NChi) fabric was picked out, washed with hot distilled water, methanol, and hexane, and then dried at room temperature.

\subsection{Dyeing experiments}

The surfaced-modified nylon 6 fabrics $(10 \mathrm{~cm} \times 10$ $\mathrm{cm}$ ) were dyed by acid dye and reactive dye, respectively. The nylon 6 fabrics were dyed by acid dye as follows. A $0.15 \mathrm{~g}$ portion of acid dye was dissolved in a $200 \mathrm{ml}$ water solution of 30vol\% acetic acid. The nylon 6 fabrics were placed in the solution, which was kept at $60{ }^{\circ} \mathrm{C}$, and 


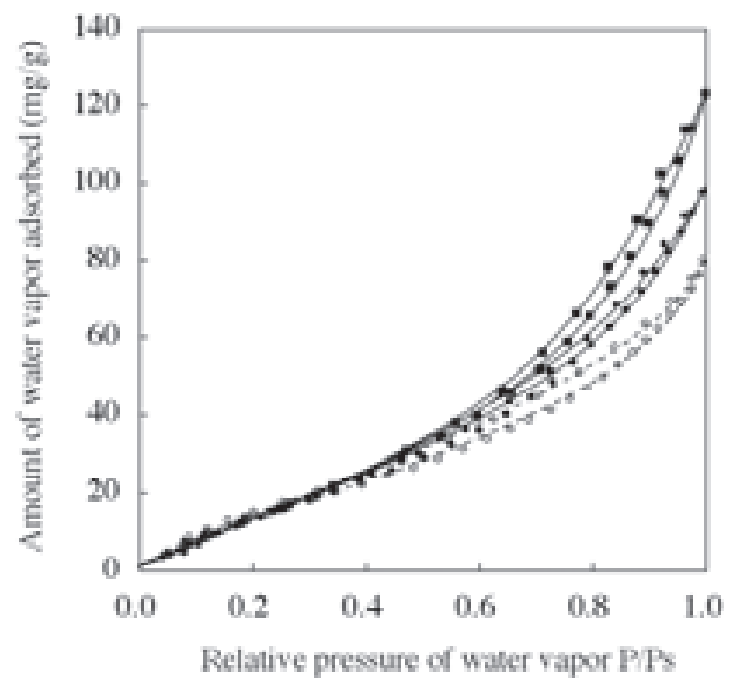

Fig. 2 Adsorption-desorption isotherm of water vapor on nylon 6 fiber $\left(25^{\circ} \mathrm{C}\right)$. $\bigcirc$ : nylon 6 , $\bigcirc$ : nylon 6 (NChi) ; 2-step reaction, $\mathbf{\square}$ : nylon 6 (NChi); one-pot method

the solution was heated until boiling. After $60 \mathrm{~min}$, the fabrics were retrieved, washed thoroughly with running tap water, and then dried at room temperature. Meanwhile, reactive dye was also used as follows. A $0.30 \mathrm{~g}$ portion of reactive dye was dissolved in a $100 \mathrm{ml} 6.6 \mathrm{mM}$ phosphate buffer solution ( $\mathrm{pH} 7.0)$. The nylon 6 fabrics were placed in the solution, which was kept at $80{ }^{\circ} \mathrm{C}$, and the solution was heated until boiling. After $40 \mathrm{~min}$, the dyed fabrics were washed thoroughly and dried by the same methods of acid dye. The dye concentration on nylon 6 fabric was estimated by K/S values using usual methods, which were calculated from their reflectivity $(550 \mathrm{~nm})$ as measured with a UV-VIS spectroscopy equipped with an integrating sphere (UV-3100A, Shimadzu Co., Japan).

\subsection{Measurement of fiber property}

\subsubsection{Hygroscopic property}

The adsorption-desorption isotherms of water vapor on the nylon 6 fabrics were measured at $25{ }^{\circ} \mathrm{C}$ with an automatic vapor adsorption apparatus (BELSORP 18S, Nihon Bell Co., Japan), with the fabric placed in a sample tube, and dried in a vacuum before measurements. In the adsorption process, water vapor was automatically added to the tube until relative vapor pressure (P/Ps) reached 0.99. In the subsequent desorption process, water vapor was gradually removed from the nylon 6 in the tube until reached 0.10. A part of the obtained isotherms are figured in Fig. 2. In this paper, the amount of water vapor in the nylon 6 fabric during the desorption process $(\mathrm{P} / \mathrm{Ps}=0.80)$ was taken as the hygroscopicity index of nylon 6 fabric and is shown below.

\subsubsection{Mechanical strength}

The mechanical strength of the nylon 6 fabric was evaluated in term of relative tensile strength measured using a tensile test machine (AGS-5kNJ, Shimadzu Co., Japan). Test pieces $(2.5 \mathrm{~cm} \times 23 \mathrm{~cm})$ were fixed with a distance of $15 \mathrm{~cm}$ between the chucks, and then were stretched at a constant speed of $200 \mathrm{~mm} / \mathrm{min}$ under the standard conditions $\left(20{ }^{\circ} \mathrm{C}, 50 \mathrm{rh} \%\right)$. The tensile strength of the original nylon 6 fabric was $143 \mathrm{~N} / \mathrm{cm}$.

\section{Results and Discussion}

\subsection{Reaction of nylon 6 fiber with cyanuric chloride (1st step reaction)}

In our previous paper, reactions of PET fibers having amino groups with hydrophobic cyanuric chloride resulted in a little decrease in their hygroscopicity and a remarkable decrease in their $\mathrm{K} / \mathrm{S}$ values for acid dyes [10]. In this work, the reaction of nylon 6 fiber with cyanuric chloride was examined on the same condition of PET fibers. Unexpectedly, both their hygroscopic property and dyeing abilities for acid dyes were not affected by this reaction (Figs. 3 and 4-a). Meanwhile, K/S value for the reactive dyes was dramatically decreased for about 15 min similar to one of the PET fibers dyed by the acid dyes (Fig. 4-b). These different results between the nylon 6 fibers and the PET fibers can be explained as following. Most amino groups of the PET fibers are introduced chemically only on their surface by reactions with diamine, whereas the amino groups of the nylon 6 fibers originally exist in their internal moiety in addition to their surface. This indicates that the reactive dyes can react with only the amino groups near the surface, but acid

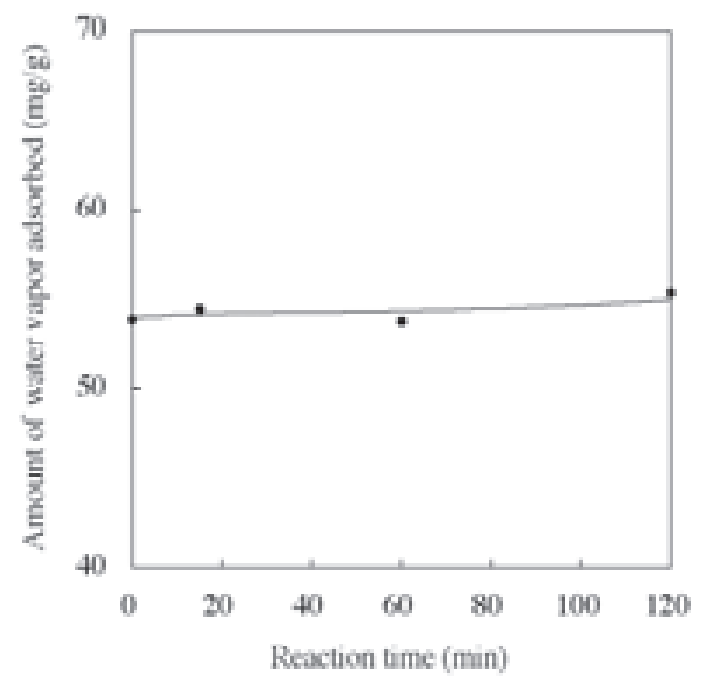

Fig. 3 Influence of reaction time with cyanuric chloride on hygroscopicity of nylon 6 fiber. 


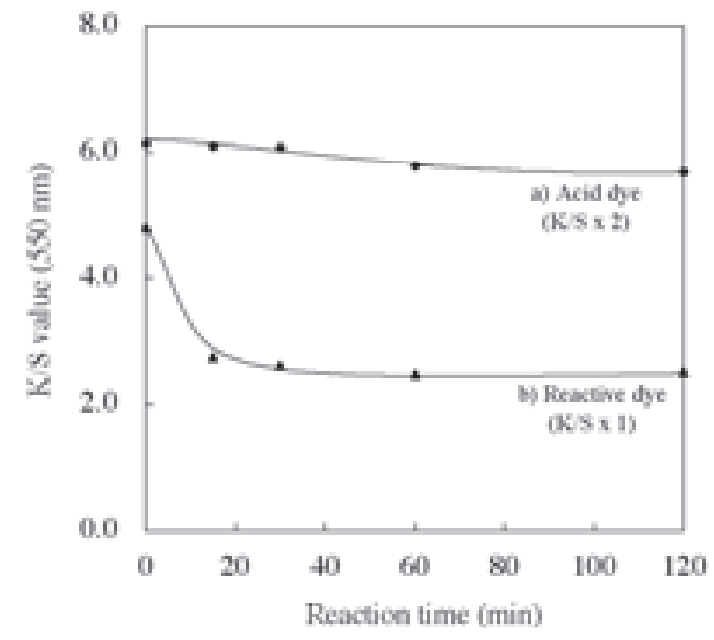

Fig. 4 Influence of reaction time with cyanuric chloride on dyeing ability of nylon 6 fiber.

dyes or water molecules can also interact with the amino groups existing in the deeper position of the nylon fibers. As a result, cyanuric chloride can react with the amino groups on the surface of the nylon 6 fibers to afford the reactive cyanuric groups on their surface, but most of other amino groups cannot participate in this reaction.

\subsection{Reaction of nylon 6 having cyanuric groups with chitosan (2nd step reaction)}

\subsubsection{Reaction temperature}

Fig. 5 shows the amounts of water vapor adsorbed on the nylon 6 fibers plotted against the reaction time with chitosan (reaction temperature: $25-80{ }^{\circ} \mathrm{C}$ ). At the temperature below $40{ }^{\circ} \mathrm{C}$, the increase of the amounts of water vapor adsorbed on the fibers was not observed, expected for a little initial increase due to protonation of the nylon 6 by acetic acid. When the reaction temperature was lower than the glass transition point $\left(T_{g}\right)$ of nylon 6 in water, the large chitosan molecules might not be able to

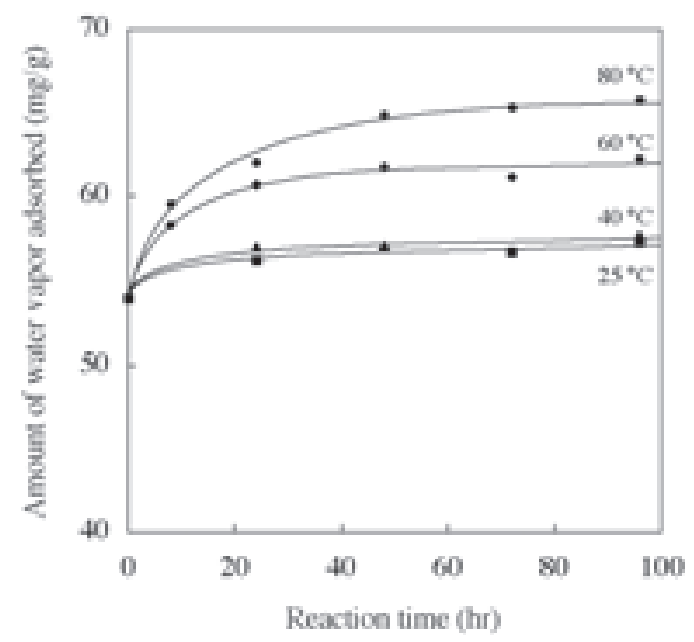

Fig. 5 Influence of reaction time with chitosan on hygroscopicity of nylon 6 fiber $([\mathrm{Chi}]=1.0 \mathrm{~g} / \mathrm{l})$. incorporate in the nylon 6 fibers to react with their cyanuric groups. At the high temperature such as 60 or $80{ }^{\circ} \mathrm{C}$, the reaction remarkably improved its hygroscopic property until the reaction time became about $48 \mathrm{hr}$. The highest enhancement of the hygroscopicity of the nylon 6 fibers was observed at the highest temperature of $80{ }^{\circ} \mathrm{C}$ $(\operatorname{Max}=65.8 \mathrm{mg} / \mathrm{g})$. Additionally, chitosan with low molecular weight hydrolyzed in the acidic reaction solutions may participate in enhancement of hygroscopic properties at these high temperature conditions.

\subsubsection{Chitosan / acetic acid concentration}

Both the chitosan and acetic acid concentration were also examined to obtain the more hydrophilic nylon 6 fibers. As a result, an increase of chitosan concentration enhanced the hygroscopicity of the fibers greatly, but the addition of chitosan at the concentration above $1.0 \mathrm{~g} / \mathrm{l}$ gradually decreased in the hygroscopicity (Fig. 6). This decrease can be explained by the chitosan gels formed at the high chitosan concentration in the acidic water solution. This gel can decrease in the fluidity of the reaction solution and prevent chitosan from being incorporated into the nylon polymer, which result in a lowering of the reactivity of chitosan toward the cyanuric groups of the fibers. On the other hand, the acetic acid concentration did not influence the hygroscopicity with exception of their very low concentration $(<2.5 \mathrm{vol} \%)$ as figured in Fig. 7. At the low concentration of acetic acid, the insolubilization of chitosan is caused by a lack of the acid components, which decreased the chitosan concentration at the same time. Thus a balance of the concentrations between chitosan and acetic acid is very important to keep the homogeneous chitosan solution to enhance their hygroscopicity.

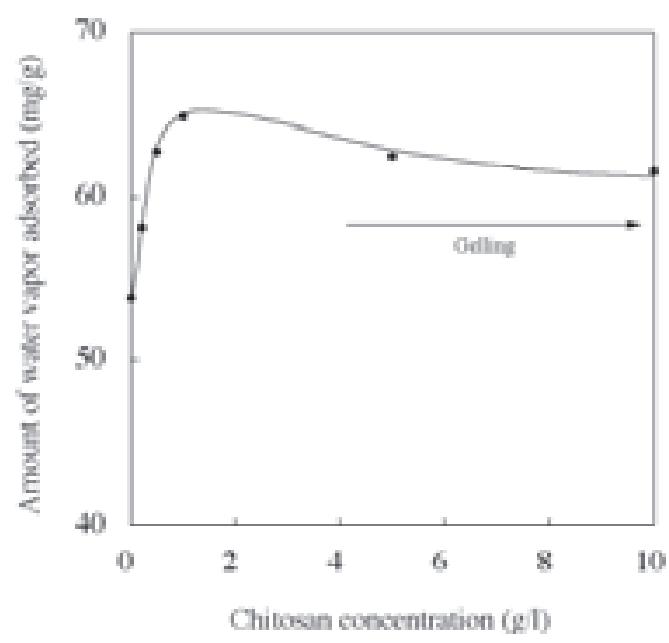

Fig. 6 Influence of chitosan concentration on hygroscopicity of nylon 6 fiber $\left(80^{\circ} \mathrm{C}, 48 \mathrm{hr},[\mathrm{AcOH}]=\right.$ $5.0 \mathrm{vol} \%)$. 


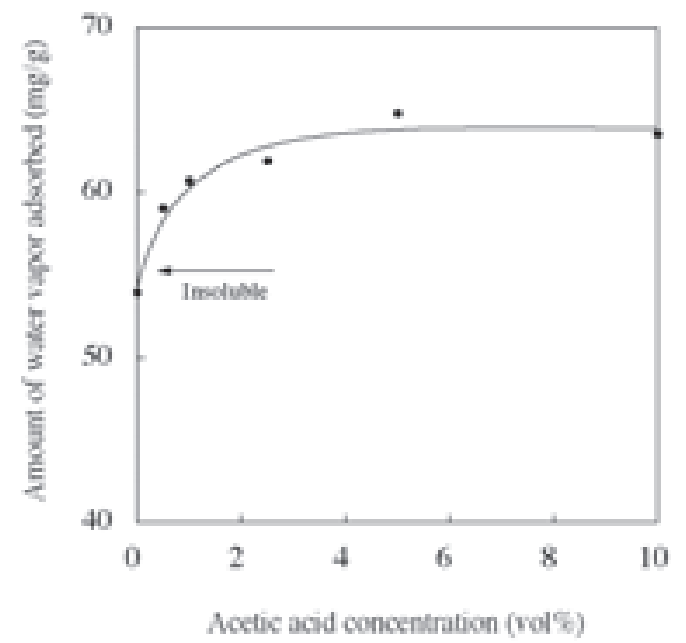

Fig. 7 Influence of acetic acid concentration on hygroscopicity of nylon 6 fiber $\left(80{ }^{\circ} \mathrm{C}, 48 \mathrm{hr}\right.$, [Chi] $=$ $1.0 \mathrm{~g} / 1)$.

\subsubsection{Mechanical strength of nylon 6 fabric modified by chitosan}

The mechanical strength of the nylon 6 fibers after the reactions with chitosan is shown in Fig. 8 (reaction temperature: $60,80{ }^{\circ} \mathrm{C}$ ). Unexpectedly, the mechanical strength was linearly decreased in proportional to the reaction time at both temperatures. Especially, the higher reaction temperature resulted in a serious lowing of their mechanical strength. This indicates that the acid hydrolysis of the nylon 6 fibers proceeds in the presence of acetic acid. In Fig. 9, the relationship between the mechanical strength and the acetic acid concentration is shown. As expected, the increase of the acetic acid concentration gave a serious decrease in their mechanical strength. Furthermore, though the acid hydrolysis of nylon 6 in the absent of chitosan was examined as a

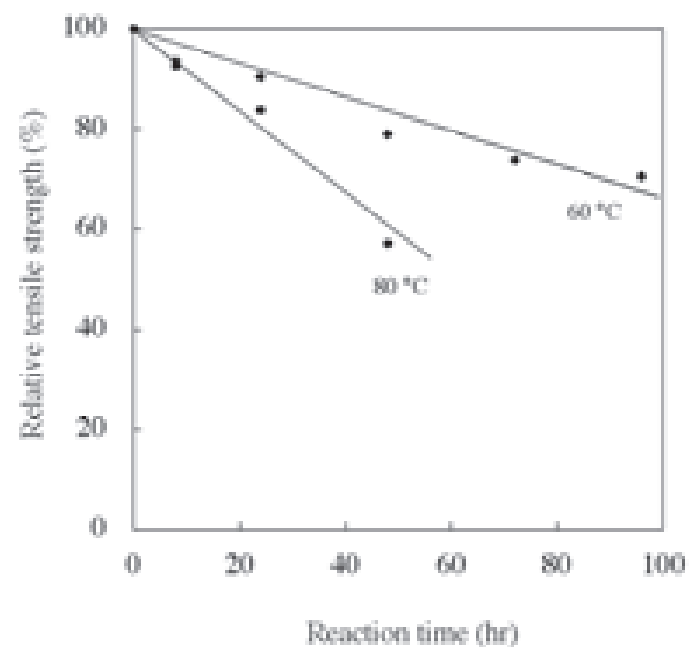

Fig. 8 Influence of reaction time with chitosan on mechanical strength of nylon 6 fiber $([\mathrm{Chi}]=1.0$ $\mathrm{g} / \mathrm{l},[\mathrm{AcOH}]=5.0 \mathrm{vol} \%)$.

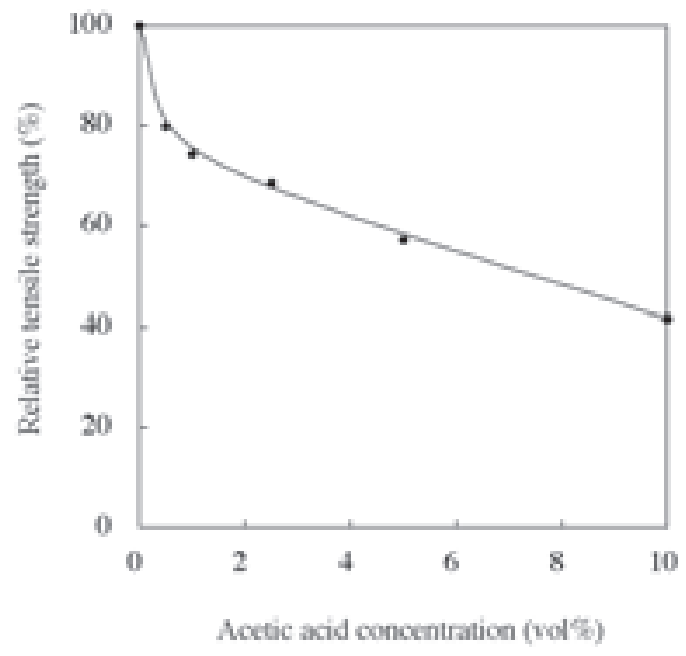

Fig. 9 Influence of acetic acid concentration on mechanical strength of nylon 6 fiber $\left(80{ }^{\circ} \mathrm{C}, 48\right.$ hr).

preliminary experiment, a large increase in their hygroscopicity was not observed (After $24 \mathrm{hr}$ in 5.0vol\% $\mathrm{AcOH}_{\text {aq }}$ at $80{ }^{\circ} \mathrm{C}, 56.6 \mathrm{mg} / \mathrm{g}$ ). This result also supports that the reactions with chitosan play an important roles in enhancement of their hygroscopicity.

\subsubsection{Dyeing ability of nylon 6 fabric modified by chitosan}

The dyeing ability of the nylon 6 after the reaction with chitosan at $80{ }^{\circ} \mathrm{C}$ was also investigated using the reactive dye (Fig. 10). Introduction of the chitosan moiety on the nylon 6 fibers afforded an increase in their $\mathrm{K} / \mathrm{S}$ values and then a saturation curve above $48 \mathrm{hr}$. This tendency agrees with the results of the hygroscopic property discussed above, because of introduction of the hydroxyl groups or the amino groups originated from chitosan. Interestingly, the $\mathrm{K} / \mathrm{S}$ values after the reaction

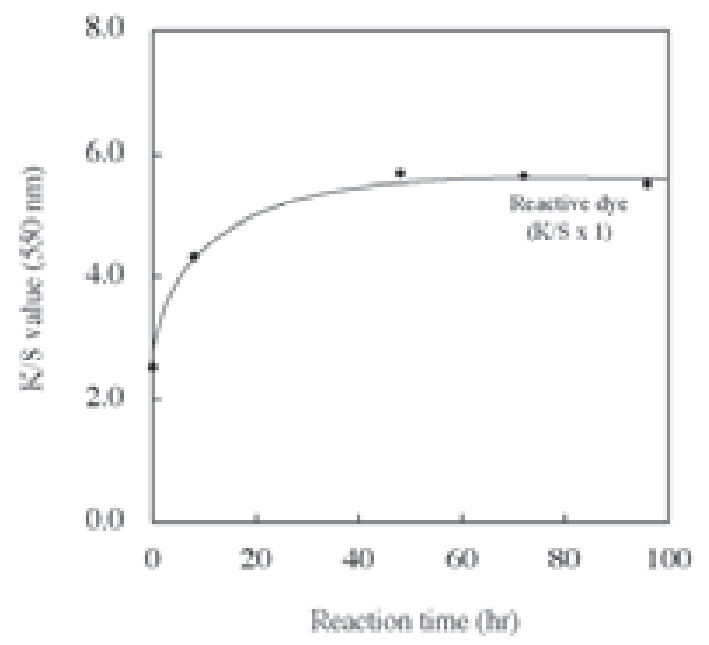

Fig. 10 Influence of reaction time with chitosan on dyeing ability of nylon 6 fiber $\left(80{ }^{\circ} \mathrm{C}\right.$, [Chi] $=$ $1.0 \mathrm{~g} / \mathrm{l})$. 
with chitosan was higher than those of the original nylon 6 fibers, that is, the nylon 6 fibers covered with the chitosan molecules can be dyed more easily than original one by reactive dyes.

\subsection{Other methods for surface modification}

\subsubsection{Reaction of nylon 6 fabric with chitosan having cyanuric groups (chitosan $(\mathrm{NCl})$ )}

Fig. 11-b shows the reaction of the nylon 6 fibers with chitosan molecules having the cyanuric groups, those were obtained from the reaction of chitosan with cyanuric chloride on the same condition of the reaction of the nylon 6 fibers. Though a part of chitosan $(\mathrm{NCl})$ was insoluble in $5.0 \mathrm{vol} \%$ acetic acid water solution, the reaction with the nylon 6 fibers was examined at the best reaction condition of the nylon $6(\mathrm{NCl})$ fibers with chitosan. As figured in Fig. 12-b, only a small initial increase of the hygroscopicity of the nylon 6 was observed similar to the reaction of the nylon $6(\mathrm{NCl})$ fiber with chitosan in 2-step reaction at the low temperature. Here, the insoluble chitosan with the hydrophobic cyanuric groups might participate in the reaction with the nylon 6 fibers.

\subsubsection{One-pot method using cationic surfactant}

We also investigated a one-pot method to react with both cyanuric chloride and chitosan simultaneously as a practical method for the industrial applications (Fig. 11-c). Cyanuric chloride is a hydrophobic organic compound and usually insoluble in water, whereas chitosan can be dissolved only in acidic water solution. Firstly, the solvents dissolving both of the compounds are needed to react in the homogeneous system, and this problem reminded us that various organic compounds could dissolve in the water solutions including surfactants. Here, the solubility tests of cyanuric chloride in acetic acid water solution containing chitosan were undergone using

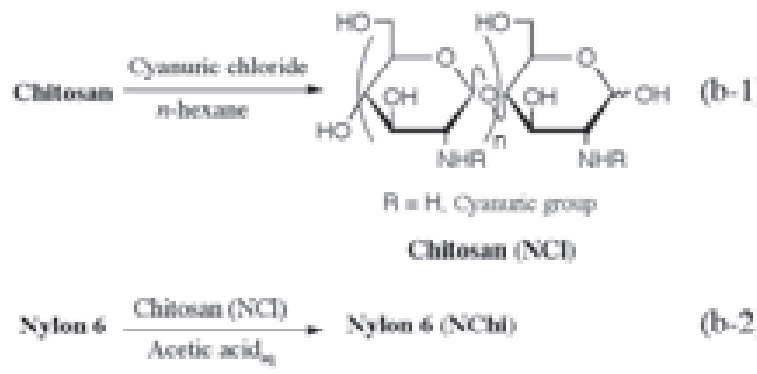

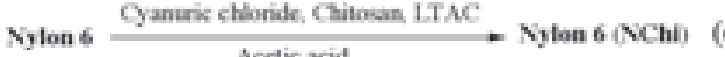

Fig. 11 Scheme of surface modification by other methods. (b) method using chitosan ( $\mathrm{NCl}$ ), (c) one-pot method.

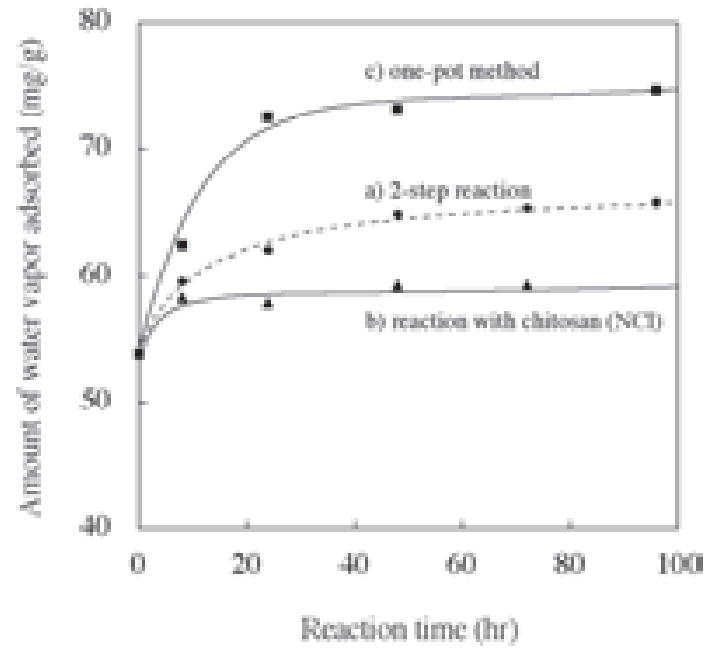

Fig. 12 Hygroscopicity of nylon 6 fiber modified by chitosan using various methods $\left(80^{\circ} \mathrm{C}\right)$.

anionic or cationic surfactants. Addition of anionic surfactant (SDS) gave the reprecipitations of chitosan from the solution, because of the formation of the insoluble ion complexes between the protonated chitosan and the anionic SDS. Meanwhile, addition of cationic surfactant (LTAC) made a clear solution including those compounds, where cyanuric chloride is incorporated into the hydrophobic environment of their micelles. Fig. 12-c shows the amounts of water vapor adsorbed on the nylon 6 fiber plotted against the reaction time using the one-pot method. Interestingly, obtained results indicated the highest hygroscopic property of the nylon 6 fiber (Max $=$ $74.7 \mathrm{mg} / \mathrm{g}$ ) in this paper. Though the detail mechanism of this one-pot method is not clear yet, cyanuric chloride will take part in not only reaction between the nylon 6 fibers and the chitosan but also between chitosan molecules as a cross-linking agent.

In our next papers, other fiber properties of these hygroscopic nylon fibers modified by chitosan such as electrostatic propensity and antibacterial activity will be investigated in addition to detail explanations of the mechanism of the one-pot method using the cationic surfactants.

\section{Conclusion}

In this work, reactions of nylon 6 fibers with cyanuric chloride and the following reaction with chitosan gave chitosan-coated nylon 6 fibers. The first reaction with cyanuric chloride decreased their dyeing ability toward reactive dye by introduction of the cyanuric groups, whereas the second reaction with chitosan improved both their dyeing ability and hygroscopic property by introduction of the chitosan 
moieties. As a result, their hygroscopicity was largely influenced by the reaction temperature, the reaction time with chitosan, and the chitosan or acetic acid concentration. Finally, a one-pot method using cationic surfactant LTAC was also investigated. As a result, above two reactions could proceed simultaneously and this method afforded the largest enhancement of the hygroscopicity. Especially, this method without organic solvents can be expected as one of the useful fiber processings toward the environmental issues.

\section{References}

1. Y. Ikushima, O. Sato, T. Yokoyama, and M. Arai, AngewChem. Int. Ed., 38, 2910 (1999).

2. M. Goto, S. Umeda, A. Kodama, T. Hirose, and S. Nagaoka, Koubunshi Ronbunshu, 58, 548 (2001).
3. S. Matsukawa, M. Kasai, and Y. Mizuta, Sen'i Gakkaishi, 51, 17 (1995).

4. T. Nakamura, Japanese Patent Disclosure, H7229024 (1995).

5. H. Shin, S. Takino, M. Ueda, and K. Suzuki, Sen'i Gakkaishi, 54, 400 (1998).

6. A. D. Pozzo, L. Vanini, M. Fagnoi, M. Guerrini, A. D. Benedittis, and R. A. A. Muzzarelli, Carbohydr. Polym., 42, 201 (2000).

7. S. Hiigaki, S. Morimoto, and R. Nagabe, Kakougijyutsu, 37, 597 (2002).

8. T. Ohe, Y. Yoshimura, and I. Abe, Sen'i Gakkaishi, 59, 139 (2003), 60, 144 (2004).

9. T. Ohe, Y. Yoshimura, and I. Abe, Kagaku to Kogyo, 79, 120 (2005).

10. T. Ohe, Y. Yoshimura, I. Abe, M. Ikeda, and Y. Shibutani, Text. Res. J., in press. 